\title{
Viscosity variation of Cholesteryl oleyl carbonate at various temperature with Acoustic parametres
}

\author{
Jatinder Pal Singh*, Rajesh Sharma \\ Department of Physics, Post-graduate government college sector-11, chandigarh, India
}

\begin{abstract}
Viscoelastic properties of liquid crystals are very important for applications like display technology. Certain molecules do not show a single phase transition from the crystalline to the liquid state on heating, but rather a series of transformations involving new phases possessing a state of aggregation intermediate bctwcen those of highly ordered crystals and of isotropic liquids. For this reason they are termed as liquid crystals $(L C)$ or mesogens. Liquid crystals can be roughly categorized as lyotropic or thennotropic liquid crystals. Heating such crystalline solids or cooling them from their isotropic liquid phase can give rise to liquid crystalline phases. Several materials exist in the LC phase at room temperature, and many other room temperature LC materials can be produced by using mixtures of compounds. All components of the mixture need not necessarily be individually capable of forming $L C$ phases in the latter case. An essential requirement for liquid crystalline or mesomorphic behaviour is that the molecules must be geometrically anisotropic in shape.

The ultrasonic velocity measurement is a unique tool in characterizing the structure and properties of the system and provides significant information on the arrangement of matter in solutions and also finds an extensive application in studying the nature of intermolecular forces. In the present work, attempts have been made to investigate the behavior of cholesteryl oleyl carbonate at various concentrations also at different temperatures. The Ultrasonic velocity, viscosity and density are measured experimentally. In order to get more information on the nature and strength of molecular interaction, we have calculated the viscosity of cholestryl oleyl carbonate is done according to the statistical calculations [19,20].
\end{abstract}

Keywords: cholesteryl oleyl carbonate, viscosity, surface tension ,Ultrasonic Velocity,

\section{Introduction}

Cholesteryl oleyl carbonate (COC) is an organic chemical, an carbonate ester of cholesterol and oleyl alcohol with carbonic acid. It is a liquid crystal material forming cholesteric liquid crystals with helical structure. It is a transparent liquid, or a soft crystalline material with melting point around $20^{\circ} \mathrm{C}$. It can be used with cholesteryl nonanoate and cholesteryl benzoate in some thermochromic liquid crystals, its
$\underline{\text { Molecular formula }}$
$\mathrm{C}_{46} \mathrm{H}_{80} \mathrm{O}_{3}$
Molar mass
$681.13 \mathrm{~g} / \mathrm{mol}$

It is used in some hair colors, make-ups, and some other cosmetic preparations.

Some Formulae of the parameters to be calculated are

The specific acoustic impedance $(Z)$ is expressed as

Surface Tension $\underset{\overline{\mathrm{S}}}{ } \rho_{\text {is }}$ is determined by the following formula:

$v=\left(S / 6.3 \times 10^{-4} \rho\right)^{2 / 3}$

The statistical mechanical approach is also useful to predict relationships between different physical properties of liquids [19-22], such as: Relation between surface tension and viscosity is

$$
\eta / \sigma=16 / 15 \sqrt{ } \mathrm{M} / \mathrm{RT}
$$

where $\boldsymbol{\sigma}$ is the surface tension of the liquid and $\boldsymbol{\eta}$ [21] is viscosity of liquid ,T is the temperature,Eq. is justified by experimental data mainly at the

melting point of liquids [19]. In the present study, we have used the ultrasonic Interferometer (Mittal Enterprises Make) for the observation of ultrasonic velocity (C) and knowing the frequency we can find out various parameters such as adiabatic compressibilitysurface tension, viscosity. And temperature variation using temperature bath. .An ultrasonic interferometer is a widely used device to determine the ultrasonic velocity in 
liquids with high precision. The principle applied in the measurement of ultrasonic velocity $(\mathrm{C})$ is based on the accurate determination of the wavelength $(\lambda)$ in the medium.

Ultrasonic wave of known frequency $(f)$ are generated by a quartz crystal fixed at the bottom of the measuring cell. These waves are reflected by movable metallic plate kept parallel to the quartz crystal. If the separation between these two (i.e. Quartz crystal and metallic plate) is exactly a whole multiple of sound wavelength, the standing waves are formed in the medium. This acoustic resonance gives a reactionary electrical response in the form of an anode current on the frequency generator deriving the crystal. If the distance between the plate and crystal is now increased and decreased and the variation is exactly one half wavelength $(\lambda / 2)$ or a multiple of it, the anode current of the frequency generator becomes maximum. By knowing the wavelength $(\lambda)$, the ultrasonic velocity can be calculated by the relation

$\mathrm{C}($ velocity $)=$ wave length $(\lambda) \times$ frequency $(f)$

Acoustical parameters were calculated using the measure values of velocity. The values of ultrasonic velocity, surface tension of cholesteryl oleyl carbonate at different temperatures $(303 \mathrm{~K}, 308 \mathrm{~K}, 313 \mathrm{~K}, 318 \mathrm{~K}$ and $323 \mathrm{~K})$ are given in Table 1.

Table 1

\begin{tabular}{|c|c|c|c|}
\hline $\begin{array}{l}\text { Abs. } \\
\text { Temp }\end{array}$ & Velocity & $\sigma$ & Eta \\
\hline $\mathrm{T}$ & $\mathrm{C}$ & Sigmax $10^{-4}$ & $\operatorname{Eta}(\boldsymbol{\eta})$ \\
\hline 303 & 1748 & 4.53 & 2.510051422 \\
\hline 304 & 1568 & 3.84 & 2.124227009 \\
\hline 305 & 1492 & 3.55 & 1.964223859 \\
\hline 306 & 1504 & 3.58 & 1.975388192 \\
\hline 307 & 1420 & 3.28 & 1.807603847 \\
\hline 308 & 1456 & 3.40 & 1.871610292 \\
\hline 309 & 1616 & 3.97 & 2.180628857 \\
\hline 310 & 1624 & 3.99 & 2.188547632 \\
\hline 311 & 1644 & 4.06 & 2.22068596 \\
\hline 312 & 1660 & 4.11 & 2.244911738 \\
\hline 313 & 1764 & 4.49 & 2.449869386 \\
\hline 314 & 1548 & 3.68 & 2.006363233 \\
\hline 315 & 1500 & 3.51 & 1.90674912 \\
\hline 316 & 1524 & 3.58 & 1.945530084 \\
\hline 317 & 1468 & 3.38 & 1.832348809 \\
\hline 318 & 1444 & 3.29 & 1.781041827 \\
\hline 319 & 1468 & 3.36 & 1.818933842 \\
\hline 320 & 1416 & 3.18 & 1.716830414 \\
\hline 321 & 1388 & 3.08 & 1.660051655 \\
\hline 322 & 1368 & 3.01 & 1.618346817 \\
\hline 323 & 1284 & 2.73 & 1.466206633 \\
\hline
\end{tabular}



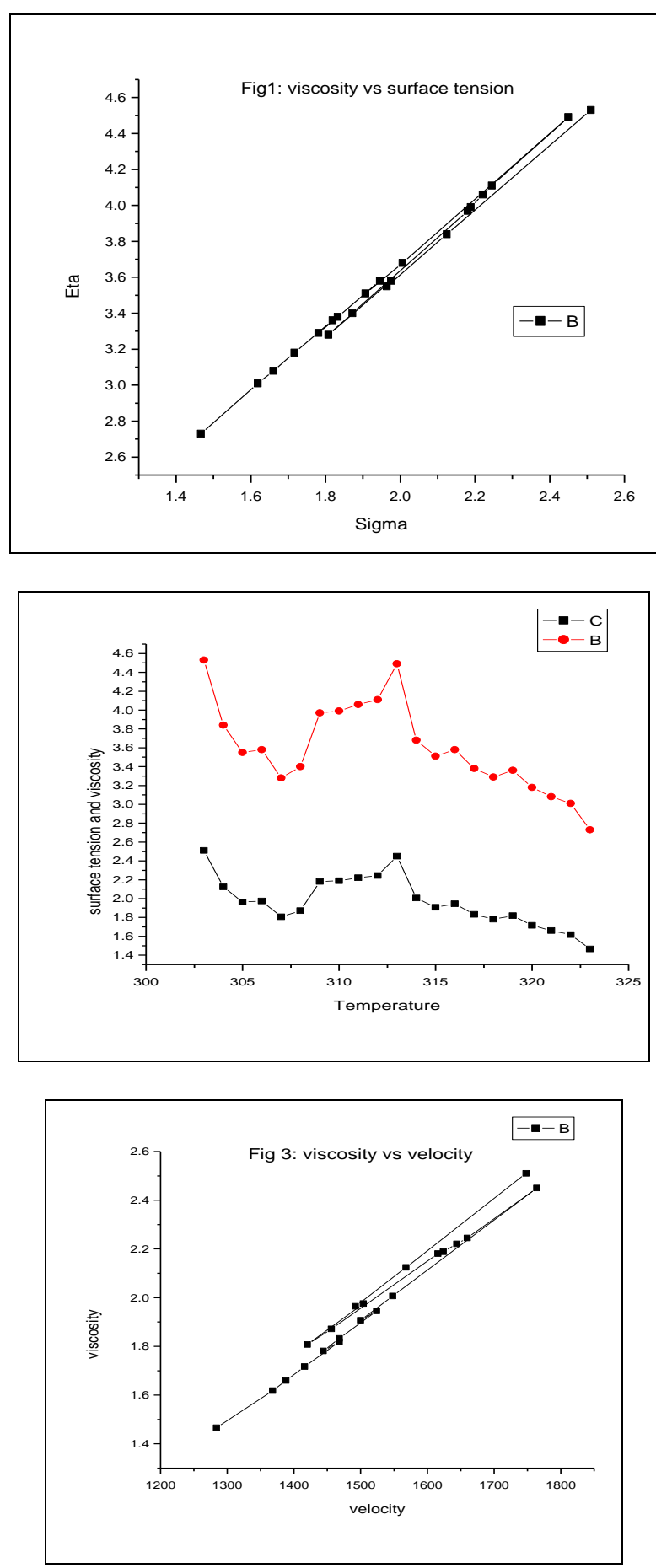

II. Results And Discussion:

In our present work, ultrasonic velocity on nonaqueous solutions of cholesteryl oleyl carbonate at different temperature were studied. The calculations of viscosity of cholestryl oleyl carbonate is done according to the statistical calculations $[19,20]$. statistical mechanics provides a solid scientific basis to calculate viscosity of liquids. One of the useful approximate solutions of the statistical mechanical approach is due to Chapman [20], who characterized the force between the atoms in liquids by a distance parameter (the Goldschmidt atomic diameter, di) and a semi-empirical energy parameter (ei).Chapman showed that the reduced experimental viscosity data can be plotted as a function of the reduced temperature using a unique curve for all liquids. From figure 1 we have concluded that surface tension and viscosity are directly proportional to each other[5,6]. Figure 2 shows variation of surface tension and viscosity with temperature. It is observed that the both surface tension and viscosity decreases with the increasing value of temperature, but a clearly visible change of the temperature evolution occurs at 307 and 313 , which may point to BP II and BP I [3] phase transitions. Recently the 
stabilization of blue phases over a temperature range of more than $60 \mathrm{~K}$ including room temperature (260$326 \mathrm{~K}$ ) has been demonstrated $[17,18]$. Highly chiral liquid crystals, on the other hand, may exhibit one or more blue phases (BP) as they are heated from the helical phase to the isotropic phase. In addition, the blue phases possess a much higher viscosity than either the helical or isotropic phase. In both cases, it is likely that the crystalline ordering of the blue phases is responsible for giving these liquids properties which one usually associates with solids. The phase of a thermotropic liquid crystal is temperature dependent. As temperature is varied, the conditions on the translational and orientational order of the constituent molecules which produce the most energetically favorable system may also change.Its very encouraging to find the values of viscosity from the surface tension calculations using ultrasonic velocity.

\section{References:}

[1] G.K. Johri, and R.C. Misra, Acustica, 67, 292 (1989)

[2] A.Ali and A.K. Nain, PRAMANA-journal of Phys1cs, 58, No.4, 695 (2002).

[3] D.C. Wright and N.D. Mermin. “Crystalline liquids: the blue phases.” Rev. Mod. Phys. 66,385 (1989).

[4] Mason W. P. (1965). Effect of Impurities and phonon processes on the ultrasonic attenuation of Germanium, Crystal Quartz and Silicon. In Physical Acoustics IIIB. W. P. Mason (Ed.). Academic Press Inc., New York, 235-285.

[5] Brugger, K. (1964). Thermodynamic definition of higher order elastic constants. Physical Review, 133., 6., A1611-A1612.

[6] P.K. Yadawa, D. Singh, D.K. Pandey \& R.R. Yadav, Elastic and acoustic properties of heavy rare-earth metals., The Open Acoustics Journal, 2., 80-86, 2009.

[7] C. S. Priya, S. Nithya, G. Velraj, A.N. Kanappan, Molecular interactions studies in liquid mixtures using ultrasonic technique, Int. J. Adv. Sci. and Tech, Vol. 18, 59-73, 2010.

[8] E. Freedman, J. Chem. Phys. 21, 1784, (1955).

[9] A.N. Kanappan and V. Rajendran, Indian J. Pure and Appl. Phys., 30, 176, (1992).

[10] V.K. Syal, Anita Chauhan and Suvarcha Chauhan, J.Pure. Appl. Ultrason., 27, pp. 61 -69 (2005)

[11] Rajesh Sharma, Study of Dielectric and Ultrasonic Properties of Organic liquids and Liquid Crystals, Ph.D. Thesis, directed by. Dr. G.K. Johri submitted to C.S.J.M. University, Kanpur, INDIA (2002).

[12] Deep Chandra Gupta, Study of the Structure of Non-spherical Polar Molecules and Their Properties, Ph.D. Thesis, directed by. Dr. G.K. Johri submitted to C.S.J.M. University, Kanpur, INDIA (1998).

[13] Mathieu Legay, Nicolas Gondrexon, Stéphane Le Person, Primius Boldo, and André Bontemps, Enhancement of Heat transfer by ultrasound: Review and recent advances, Int. J. Chem. Engg., Vol.2011, Article ID 670108.

[14] M. Nabeel Rashin and J. Hemalatha, Acoustical study on the interaction of coconut oil based copper oxide nanofluid, World Academy of Science, Engg. And Tech., Vol.64, 2012, pp. 201-205.

[15] S.Sh. Hosseini, Adam N.M. and Goudarzi K., Effect of temperature increasing on nanofluid structure, Australian J. Basic and App. Sci. Vol. 5(9), 2011, pp.979-984.

[16] R.R. Yadav, A.K. Gupta, S.K. Kor, and S. Ram, Ultrasonic properties in Au nanoparticles reinforced PVA

[17] S. Meiboom, J. P. Sethna, P. W. Anderson and W. F. Brinkman, "Theory of Blue Phase of Cholesteric Liquid Crystals." Phys. Rev. Lett. 46, 1216 (1981)

[18] P. P. Crooker ,(1989): "Plenary Lecture. The blue phases. A review of experiments." LiquidCrystals, 5:3, 751-775 solution, Vol. 10. No. 4, 2007, pp. 398-401.

[19] G. Kaptay: A unified equation for the viscosity of pure liquid metals MK_mk_2005_01_18080 - 2.12.04/druckhaus

[20] T.W. Chapman: AIChE J. 12 (1966) 395

[21] Y.S. Touloukian, S.C. Sexena, P.Hestermans: ThermophysicalProperties of Matter, Vol.11, Viscosity, IFI/Plenum, New-York,1975

[22] T. Iida, R.I.L. Guthrie: The Physical Properties of Liquid Metals,Clarendon Press, Oxford, 1993.

[23] E.N. Andrade: Phil.Mag. 17 (1934) 497. 